\title{
Coronary arterial microfistulae with meandering dilated coronary arteries and noncompaction-like myocardium
}

\author{
Hideaki Yuki ${ }^{1}$, Yuichiro Arima ${ }^{2}$, Daisuke Utsunomiya ${ }^{1}$, Koichiro Fujisue ${ }^{2}$, Masafumi Kidoh ${ }^{1}$, \\ Seitaro Oda ${ }^{1}$, Takeshi Nakaura ${ }^{1}$, Yasuyuki Yamashita ${ }^{1}$, Kenichi Tsujita ${ }^{2}$ \\ ${ }^{1}$ Diagnostic Radiology, Faculty of Life Sciences, Kumamoto University, Kumamoto, Japan \\ ${ }^{2}$ Cardiovascular Medicine, Faculty of Life Sciences, Kumamoto University, Kumamoto, Japan
}

A 40-year-old woman with repetitive palpitations and faintness was referred for the evaluation of coronary artery and myocardial disease. Coronary computed tomography angiography was performed, and volume-rendering image demonstrated significantly dilated and tortuous coronary arteries without stenosis (Fig. 1A). Intramyocardial enlarged vessels and transmural linear enhancement suggested coronary fistulae in the left ventricle (LV) (Fig. 1B, C), which was confirmed by conventional angiography (Fig. 1D). Meandering and dilation of the coronary arteries may be associated with multiple coronary artery-to-LV fistulae, which increase the blood flow and pressure in the coronary arteries. Coronary computed tomography angiography also showed endocardial-side "spongy myocardium" mimicking noncompaction with a maximal noncompaction-tocompaction ratio of 2.0 (Fig. 1 C, asterisks). Coronary fistulae and LV noncompaction are rare abnormalities of the heart with a prevalence of reportedly $0.3-0.8 \%$ and $0.24 \%$, respectively. Myocardial-endocardial gene signaling is responsible for both the development of LV myocardium and coronary vessel formation in the inner myocardial wall. It was speculated that these disorders might potentially be due to an abnormal arrest of the embryonic endomyocardial morphogenesis, although further experimental and clinical studies analyzing the relationship of these disorders should be conducted.

According to available research, this is the first reported case describing a patient whose coronary computed tomography features, i.e. meandering and dilation of coronary artery and transmural linear enhancement, helped identify the coexistence of coronary artery-to-LV microfistulae and LV noncompaction-like "spongy" myocardium.

Conflict of interest: None declared

Address for correspondence: Daisuke Utsunomiya, MD, Cardiovascular Medicine, Faculty of Life Sciences, Kumamoto University, 1-1-1, Honjo, Chuo-ku, Kumamoto-shi, Kumamoto, Japan, 860-8556, tel: +81 96373 5261, fax: +81 96373 5342, e-mail: utsunomi@kumamoto-u.ac.jp 


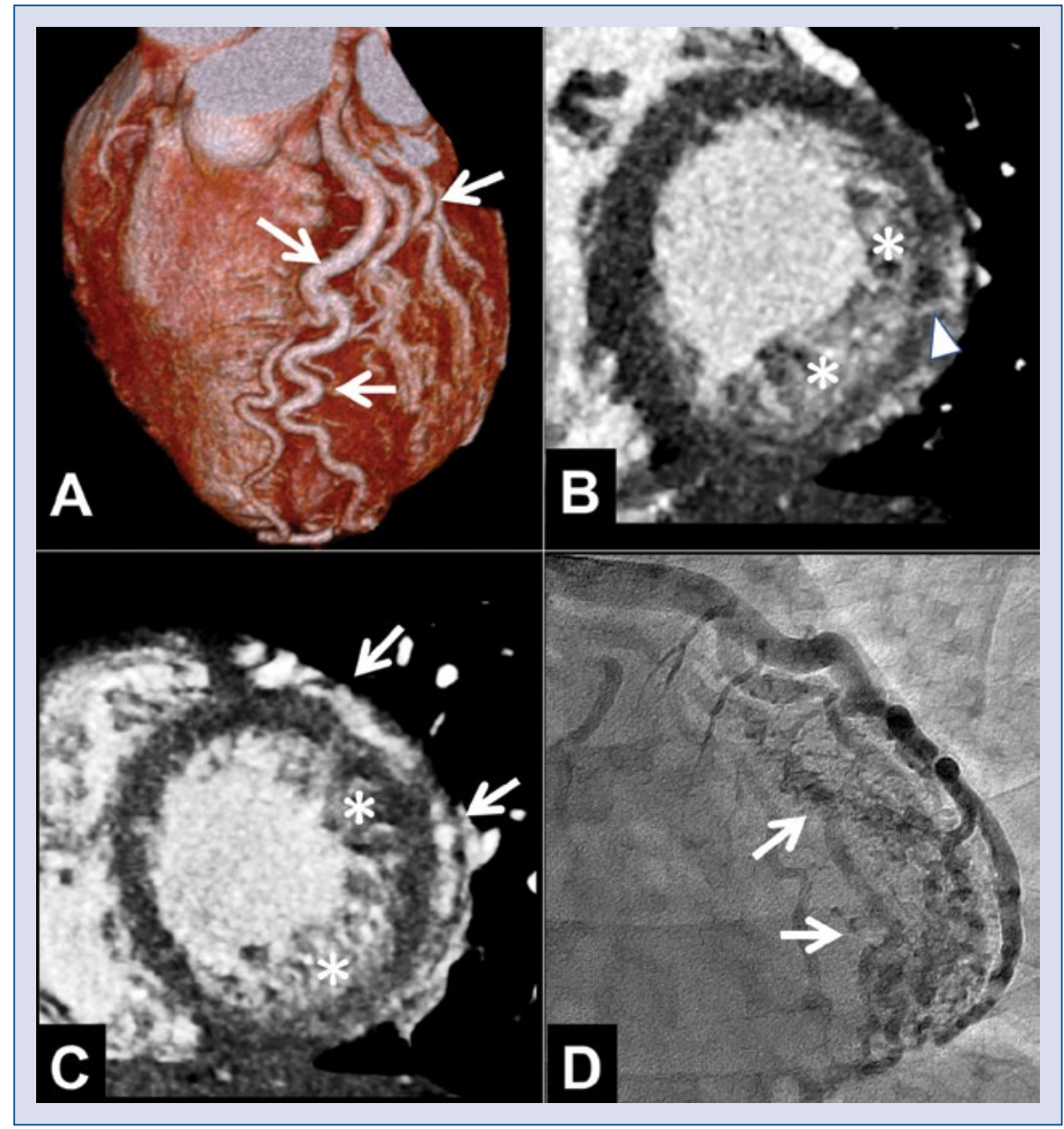

Figure 1. A. Volume-rendering computed tomography showing dilated and tortuous left coronary artery (arrows); B, C. Short-axis computed tomography images showing coronary artery-left ventricular fistulae (arrowhead) with dilated left coronary arteries (arrows) and noncompaction-like myocardium (a maximal noncompaction-to-compaction ratio of 2.0; asterisks); D. Conventional coronary angiography showing multiple coronary microfistulae (arrows). 